\title{
Diuretic properties of Orthosiphon stamineus Benth.
}

\begin{abstract}
Ethnopharmacological relevance: Orthosiphon stamineus has been used in traditional medicine for centuries especially to treat diseases of the urinary system. Aim of the study: To investigate the diuretic activity, to elucidate its possible mechanism and to evaluate the renal effects of Orthosiphon stamineus extract. Materials and methods: Water extracts were administered orally at doses of 5 and $10 \mathrm{mg} / \mathrm{kg}$ to Sprague-Dawley rats and the control groups were given commercial diuretic drugs either furosemide or hydrochlorthiazide at 10 $\mathrm{mg} / \mathrm{kg}$. Urine volume, urine $\mathrm{pH}$, urine density and urine electrolytes were determined every hour for $4 \mathrm{~h}$. Blood was assayed for glucose, albumin, blood urea nitrogen (BUN) and creatinine. Results: O. stamineus extract exhibited dose-dependent diuretic activity. However, excretion of $\mathrm{Na}+$ and $\mathrm{Cl}$ - was not markedly elevated, but urinary excretion of $\mathrm{K}+$ was significantly increased. O. stamineus extracts slightly increased the serum BUN, creatinine and blood glucose level. Although these levels were statistically significant when compared to control, these levels were still within normal range. Conclusions: O. stamineus exhibited diuretic activity, but was less potent than furosemide and hydrochlorothiazide. Care should be taken when consuming this herb as slight increase of kidney function enzymes was recorded.
\end{abstract}

Keyword: Diuretic; Electrolytes; Ortosiphon stamineus. 